\title{
A Novel MRI Biomarker of Spinal Cord White Matter Injury: T2*-Weighted White Matter to Gray Matter Signal Intensity Ratio
}

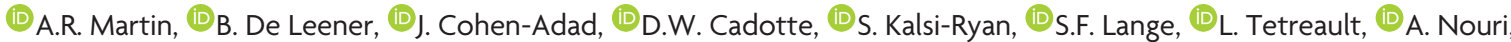 \\ (1)A. Crawley, DD.J. Mikulis, 1 H. Ginsberg, and 1 M.G. Fehlings
}

\begin{abstract}
BACKGROUND AND PURPOSE: T2*-weighted imaging provides sharp contrast between spinal cord GM and WM, allowing their segmentation and cross-sectional area measurement. Injured WM demonstrates T2*WI hyperintensity but requires normalization for quantitative use. We introduce T2*WI WM/GM signal-intensity ratio and compare it against cross-sectional area, the DTI metric fractional anisotropy, and magnetization transfer ratio in degenerative cervical myelopathy.
\end{abstract}

MATERIALS AND METHODS: Fifty-eight patients with degenerative cervical myelopathy and 40 healthy subjects underwent $3 T$ MR imaging, covering $\mathrm{Cl}-\mathrm{C} 7$. Metrics were automatically extracted at maximally compressed and uncompressed rostral/caudal levels. Normalized metrics were compared with $t$ tests, area under the curve, and logistic regression. Relationships with clinical measures were analyzed by using Pearson correlation and multiple linear regression.

RESULTS: The maximally compressed level cross-sectional area demonstrated superior differences $\left(P=1 \times 10^{-13}\right)$, diagnostic accuracy (area under the curve $=0.890$ ), and univariate correlation with the modified Japanese Orthopedic Association score (0.66). T2*WI WM/GM showed strong differences (rostral: $P=8 \times 10^{-7}$; maximally compressed level: $P=1 \times 10^{-11}$; caudal: $P=1 \times 10^{-4}$ ), correlations (modified Japanese Orthopedic Association score; rostral: -0.52 ; maximally compressed level: -0.59 ; caudal: -0.36 ), and diagnostic accuracy (rostral: 0.775; maximally compressed level: 0.860; caudal: 0.721), outperforming fractional anisotropy and magnetization transfer ratio in most comparisons and cross-sectional area at rostral/caudal levels. Rostral T2*WI WM/GM showed the strongest correlations with focal motor $(-0.45)$ and sensory $(-0.49)$ deficits and was the strongest independent predictor of the modified Japanese Orthopedic Association score $(P=.01)$ and diagnosis $(P=.02)$ in multivariate models $\left(R^{2}=0.59, P=8 \times 10^{-13}\right.$; area under the curve $=0.954$, respectively).

CONCLUSIONS: T2*WI WM/GM shows promise as a novel biomarker of WM injury. It detects damage in compressed and uncompressed regions and contributes substantially to multivariate models for diagnosis and correlation with impairment. Our multiparametric approach overcomes limitations of individual measures, having the potential to improve diagnostics, monitor progression, and predict outcomes.

ABBREVIATIONS: $\mathrm{AUC}=$ area under the curve; $\mathrm{CSA}=$ cross-sectional area; $\mathrm{DCM}=$ degenerative cervical myelopathy; $\mathrm{FA}=$ fractional anisotropy; $\mathrm{MCL}=$ maximally compressed level; $\mathrm{mJOA}=$ modified Japanese Orthopedic Association; MT = magnetization transfer; MTR = magnetization transfer ratio; qMRI = quantitative $\mathrm{MRI}$; $\mathrm{SC}=$ spinal cord; $\mathrm{SCl}=$ spinal cord injury; $\mathrm{UE}=$ upper extremity

Q uantitative MR imaging (qMRI) techniques have the potential to provide in vivo measurement of specific tissue properties, including characterizing aspects of spinal cord (SC) microstructure and tissue injury. ${ }^{1,2}$ However, efforts to apply qMRI in

Received October 20, 2016; accepted after revision January 29, 2017.

From the Division of Neurosurgery, Department of Surgery (A.R.M., D.W.C., S.K.-R., L.T., A.N., H.G., M.G.F.), and Department of Medical Imaging (A.C., D.J.M.), University of Toronto and the University Health Network, Toronto, Ontario, Canada; Polytechnique Montreal (B.D.L., J.C.-A.), Montreal, Quebec, Canada; Functional Neuroimaging Unit (J.C.-A.), Centre de recherche de l'Institut universitaire de gériatrie de Montréal, Université de Montréal, Montreal, Quebec, Canada; and University of Groningen (S.F.L.), Groningen, the Netherlands. clinical studies have thus far achieved only modest success. ${ }^{3}$ The strongest results include cross-sectional area (CSA) as a measure of spinal cord atrophy, the DTI metric fractional anisotropy (FA) to evaluate axonal integrity, and the magnetization transfer ratio (MTR) as a measure of demyelination. ${ }^{3}$ Spinal cord CSA has shown moderate-to-strong correlation with disability in $\mathrm{MS}^{4-6}$ but is a nonspecific measure of tissue injury and shows high intersubject variability in healthy subjects, ${ }^{7,8}$ somewhat limiting its

Please address correspondence to Michael G. Fehlings, MD, SCI-CRU, 11th Floor, Toronto Western Hospital, 399 Bathurst St, Toronto, M5T 2S8, ON, Canada; e-mail: michael.fehlings@uhn.on.ca, madeleineoh@gmail.com; @DrFehlings

- - Indicates open access to non-subscribers at www.ajnr.org

http://dx.doi.org/10.3174/ajnr.A5162 


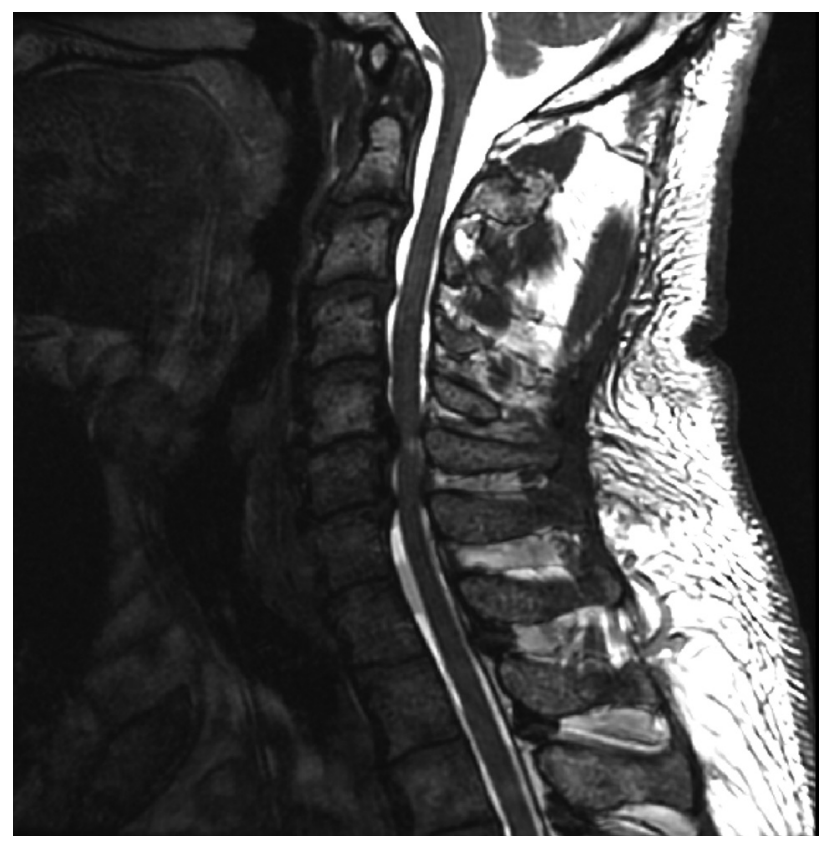

FIG 1. T2WI showing a subject with DCM with spinal cord compression. Sagittal T2WI in a subject with DCM with severe impairment shows multilevel disc degeneration, spondylosis, and spinal cord compression at C5-6 with focal hyperintensity.

utility. FA has demonstrated moderate correlation with global and focal disability in dozens of studies involving various pathologies $^{3,9-14}$ but has yet to achieve clinical uptake due to a lack of standardized/portable acquisition methods and cumbersome analysis techniques. MTR has also shown correlation with impairment in MS and spinal cord injury (SCI) studies, ${ }^{15-17}$ but results have been inconsistent, in part due to T1 and frequency offset dependencies, and thus insufficient to drive clinical adoption. ${ }^{3}$

At $3 \mathrm{~T}$ or higher field strengths, $\mathrm{T} 2^{*}$-weighted imaging of the SC provides high resolution and strong contrast between GM and WM, allowing segmentation between these structures and calculation of their CSA. ${ }^{18}$ It has also been established that $\mathrm{T} 2{ }^{\star} \mathrm{WI}$ shows hyperintensity in injured SC WM in various pathologic conditions. ${ }^{19,20}$ We hypothesized that T2*WI hyperintensity is a general phenomenon in WM injury, leading to decreased graywhite contrast that can be quantified by normalizing the WM signal intensity within each axial section by that of the GM as a $\mathrm{T} 2{ }^{*} \mathrm{WI} W M / G M$ signal-intensity ratio. Our investigation in 40 healthy subjects established that T2*WI WM/GM has lower intersubject variability compared with CSA, FA, and MTR and superior reliability compared with FA and MTR, ${ }^{7}$ though the latter metrics showed acceptable results, in keeping with prior reports. ${ }^{11,21-25}$

These encouraging findings prompted the current study in degenerative cervical myelopathy (DCM), a common condition involving degeneration of the discs, ligaments, and vertebrae, resulting in cervical spinal cord compression and functional impairment (Fig 1). ${ }^{26,27}$ We aimed to determine how well T2*WI WM/GM differs between patients with DCM and healthy subjects and correlates with global disability and focal neurologic deficits when extracted from corresponding regions of WM, in comparison with FA, MTR, and CSA of the SC.

\section{MATERIALS AND METHODS Study Design and Subjects}

This study received institutional approval from the University Health Network (Toronto, Ontario, Canada), and all participants provided written informed consent. Fifty-eight patients with DCM were consecutively recruited from the outpatient spine neurosurgery clinic, and 42 healthy subjects were recruited between October 2014 and December 2016. Patients with DCM with confounding neurologic impairment, such as diabetic neuropathy or symptomatic lumbar radiculopathy, were excluded. All subjects were examined by an experienced physician (M.G.F, A.R.M.). Two subjects recruited as healthy volunteers were found to have clinical and imaging evidence of mild DCM and were analyzed as subjects with DCM. Two subjects with DCM failed to complete the MR imaging study due to pain/claustrophobia and were excluded from analysis. Thus, 58 patients with DCM and 40 healthy subjects for analysis remained. DCM severity was categorized on the basis of the modified Japanese Orthopedic Association $(\mathrm{mJOA})$ score $($ normal $=18$ points) into mild $(\mathrm{mJOA}=$ $15-17)$, moderate $(\mathrm{mJOA}=12-14)$, and severe $(\mathrm{mJOA}<12){ }^{26}$ Three patients with DCM had undergone previous cervical operations with metallic implants and had achieved a complete or near-complete recovery $(\mathrm{mJOA} \geq 17)$ followed by new cord compression at another cervical level.

\section{Clinical Assessments}

Subjects with DCM were assessed with the following: 1) the mJOA score to determine overall functional impairment; 2) the International Standards for Neurologic Classification of Spinal Cord Injury upper extremity (UE) motor score consisting of power testing (5-point score) in 10 upper extremity muscle groups (maximum score $=50$ ) on both sides $^{28}$; and 3 ) the UE sensory score consisting of Semmes Weinstein monofilament testing in C6, C7, and C8 dermatomes (4 points each, maximum score = 12). Healthy subjects all had $\mathrm{mJOA}=18$ and were assumed to have full motor (50/50) and sensory (12/12) scores for analyses.

\section{MR Imaging Acquisitions}

Subjects underwent high-resolution isotropic T2WI, DTI with single-shot EPI, spoiled gradient-echo imaging with and without magnetization transfer (MT) prepulse, and $\mathrm{T} 2{ }^{\star} \mathrm{WI}$ with multiecho recombined gradient-echo at 3T (Signa Excite HDxt; GE Healthcare, Milwaukee, Wisconsin) as described in a companion article. ${ }^{7}$ The multiecho recombined gradient-echo sequence uses 3 echoes that are magnitude-reconstructed and combined with a sum-of-squares algorithm. Total imaging time was approximately $30-35$ minutes, including subject positioning, section prescription, and second-order localized shimming.

\section{Image-Analysis Techniques}

Template-based analysis was performed by using the Spinal Cord Toolbox, Version 2.3 (https://sourceforge.net/projects/spinal cordtoolbox/), ${ }^{29}$ as described in the companion article. ${ }^{7}$ Metrics included CSA from T2WI, FA, MTR, and T2*WI WM/GM signalintensity ratio extracted from the rostral uncompressed SC (C1$\mathrm{C} 3$ ); the maximally compressed level (MCL); and the caudal un- 


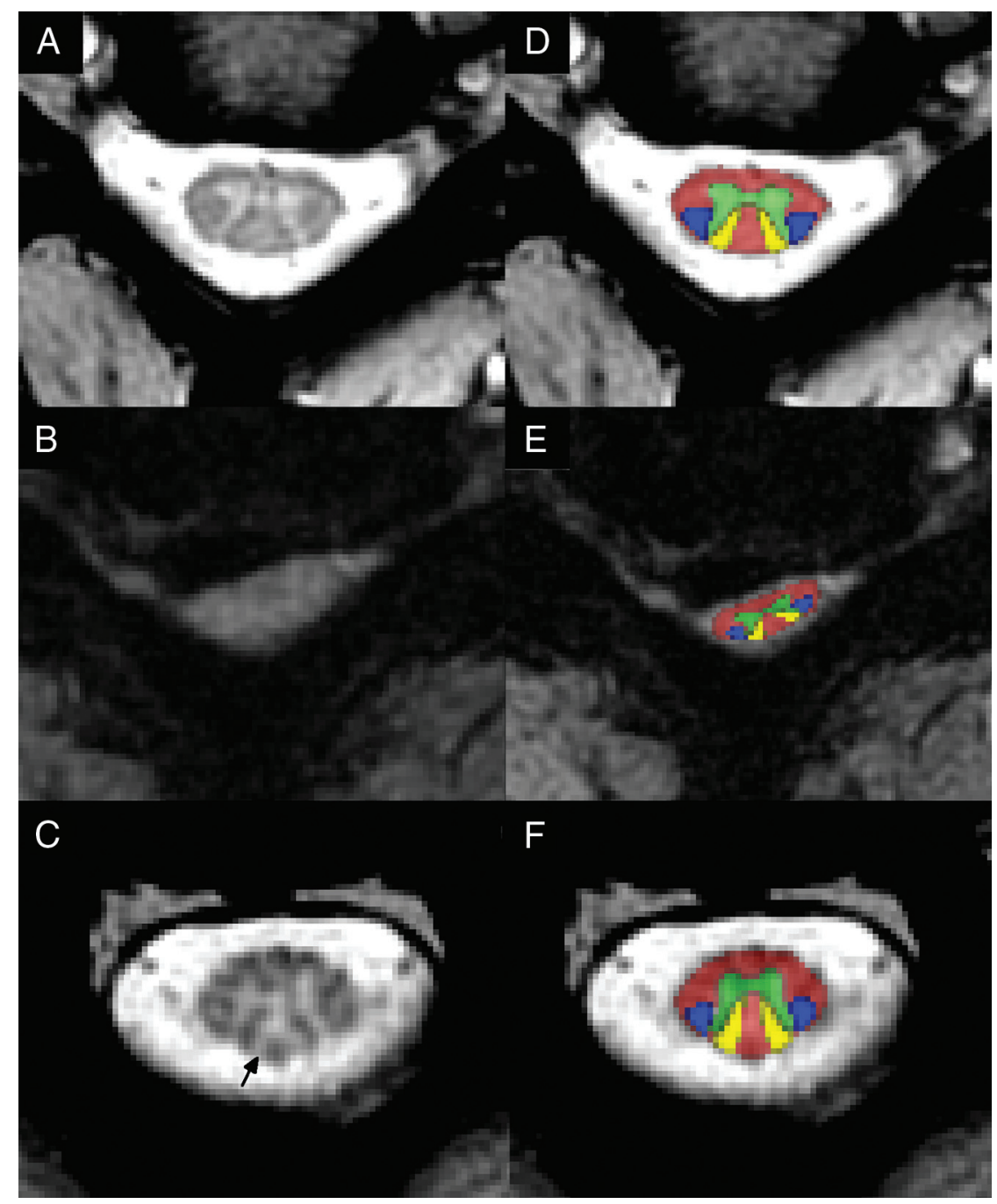

FIG 2. $\mathrm{T} 2 * \mathrm{WI}$ demonstrating loss of gray-white contrast and Wallerian degeneration. $A$, Axial $\mathrm{T} 2 * \mathrm{WI}$ at $\mathrm{C} 3-4$ in a healthy subject shows strong contrast between GM and WM (T2*WI WM) $\mathrm{GM}=0.791$ for this image). $B, \mathrm{~T} 2{ }^{*} \mathrm{Wl}$ at $C 5-6$ in a subject with severe DCM shows SC compression from a lateral disc herniation, with loss of gray-white contrast (T2*WI WM/GM $=0.967$ ). $C, T 2 * W I$ at C3 in the same subject with DCM shows focal hyperintensity (arrow) within the dorsal columns, suggesting Wallerian degeneration $(T 2 * W I W M / G M=0.923) . D-F$, The same images as in $A-C$ with the Spinal Cord Toolbox probabilistic atlas representations of WM (red), GM (green), lateral corticospinal tracts (blue), and fasciculus cuneati (yellow) overlaid.

compressed cord (C6-C7). For MCL metrics, CSA was extracted from a single section, whereas FA, MTR, and T2*WI WM/GM were averaged over 3 sections centered at the compressed level. In subjects with motion artifacts on the T2WI, T2*WI was used to calculate the CSA with correction for the oblique angle. For FA, MTR, and T2*WI, ROIs included total WM, GM (T2*WI only), and the left/right fasciculus cuneatus and lateral corticospinal tract (Fig 2). Sagittal and reformatted axial T2WI were visually assessed for SC hyperintensity by 2 raters (A.R.M., A.N.), with disagreements resolved by discussion.

\section{Statistical Analysis}

Statistical analysis was performed with $\mathrm{R}$ statistical and computing software, Version 3.3 (http://www.r-project.org). Metrics are reported as mean $\pm \mathrm{SD}$. Comparisons between characteristics of healthy subjects and those with DCM were made by using 2sample $t$ tests and $\chi^{2}$ tests. MR imaging metrics were normalized to correct for confounding relationships according to the following linear equations, developed from data in 40 healthy subjects ${ }^{7}$ :

1) $\mathrm{CSA}_{\text {corrected }}=\mathrm{CSA}_{\text {raw }}-5.3690$

$\times($ Cervical Cord Length -10.6$)$,

2) $\mathrm{FA}_{\text {corrected }}=\mathrm{FA}_{\text {raw }}+0.0012053$

$$
\times(\text { Age }-47.1) \text {, }
$$

3) $\mathrm{MTR}_{\text {corrected }}=\mathrm{MTR}_{\text {raw }}+0.17410$

$$
\times(\text { Height }-171.6)+0.074131
$$

$$
\times(\text { Age }-47.1) \text {. }
$$

In Equation 1, CSA is in square millimeters and cervical cord length is in centimeters; in Equation 2, age is in years; and in Equation 3, MTR is expressed as a percentage, height is in centimeters, and age is in years. Metrics were then converted to $z$ scores to normalize across rostrocaudal levels (eg, for comparisons at the MCL). Comparisons of normalized MR imaging metrics between DCM and healthy subjects were made by using Welch $t$ tests. These tests were also repeated against an agematched group (by excluding healthy subjects younger than 40 years of age) to confirm the findings. Diagnostic accuracy was assessed with the area under the curve (AUC) and logistic regression, with backward stepwise variable selection. Relationships between normalized MR imaging metrics and clinical measures were assessed by using Pearson correlation coefficients and backward stepwise multiple linear regression. CSA of the SC and other metrics extracted from the total WM were analyzed against the mJOA score, while metrics from each lateral corticospinal tract and fasciculus cuneatus were analyzed against ipsilateral UE motor and sensory scores, respectively. Two-way ANOVA with an interaction term was used to assess how T2* WI WM/GM and T2WI hyperintensity relate to the mJOA score. Results were considered statistically significant at $P<.05$, due to the exploratory nature of this study.

\section{RESULTS}

\section{Subject Characteristics}

Subjects with DCM showed the following distribution of severity: 33 mild, 15 moderate, and 10 severe. Age differed significantly between healthy subjects and those with DCM (mean, $47.1 \pm 15.3$ versus $57.0 \pm 10.9$ years, $P=3 \times 10^{-4}$; Table 1$)$. When healthy subjects younger than 40 years of age were excluded, age became equivalent ( $n=26$; mean age, $56.3 \pm 9.8$ years, $P=.76$ ). Other 
demographic variables (sex, height, weight, and neck length) did not vary between groups.

\section{Image Acquisition and Analysis}

Four T2WI datasets and 1 T2*WI dataset were excluded due to motion artifacts. Individual sections were excluded due to artifacts as follows: DTI: 5.3\%; MT: 0.8\%; and T2^WI: 0.7\%. Three patients with metallic implants had images excluded at those levels and 2 axial sections above and below them; the remaining images and metrics appeared to be of acceptable quality. Analysis of subjects with DCM required manual editing of segmentation masks in most cases due to deformation of the cord and a lack of contrast with surrounding tissues, requiring $<5$ minutes per dataset. Automatic registration to the Spinal Cord Toolbox template/atlas was successful in all cases.

\section{MR Imaging Metrics}

Significant differences between DCM and healthy subjects were found in 10/12 MR imaging metrics (Table 2), including decreased CSA (rostral: $P=9 \times 10^{-5}$; MCL: $P=1 \times 10^{-13}$ ),

Table 1: Subject characteristics ${ }^{\mathrm{a}}$

\begin{tabular}{lcc}
\hline \multicolumn{1}{c}{ Characteristic } & $\begin{array}{c}\text { Healthy } \\
\text { Subjects } \\
(\boldsymbol{n}=\mathbf{4 0})\end{array}$ & $\begin{array}{c}\text { Subjects } \\
\text { with DCM } \\
(\boldsymbol{n}=\mathbf{5 8})\end{array}$ \\
\hline Age (yr) & $47.1 \pm 15.3$ & $57.0 \pm 10.9^{\mathrm{b}}$ \\
Sex (M/F) & $21: 19$ & $36: 22$ \\
Height $(\mathrm{cm})$ & $171.4 \pm 8.6$ & $172.4 \pm 10.4$ \\
Weight $(\mathrm{kg})$ & $74.6 \pm 11.5$ & $74.9 \pm 9.9$ \\
Neck length (mm) & $106.1 \pm 9.6$ & $106.8 \pm 9.4$ \\
mJOA & $18.0 \pm 0.0$ & $14.2 \pm 2.5^{\mathrm{b}}$ \\
R UE motor & $50.0 \pm 0.0^{\mathrm{c}}$ & $46.1 \pm 5.2^{\mathrm{b}}$ \\
L UE motor & $50.0 \pm 0.0^{\mathrm{c}}$ & $46.5 \pm 5.6^{\mathrm{b}}$ \\
R UE sensation & $12.0 \pm 0.0^{\mathrm{c}}$ & $10.5 \pm 2.5^{\mathrm{b}}$ \\
L UE sensation & $12.0 \pm 0.0^{\mathrm{c}}$ & $10.6 \pm 2.5^{\mathrm{b}}$ \\
\hline
\end{tabular}

Note:- L indicates left; R, right.

${ }^{a}$ Demographics and clinical measures are reported as mean \pm SD.

b Significant differences $(P<.05)$ between those with DCM and healthy subjects.

c Motor and sensory scores for healthy subjects were assumed to be full, on the basis of a screening examination.

Table 2: Summary of MRI metrics ${ }^{\mathrm{a}}$

\begin{tabular}{|c|c|c|c|c|}
\hline $\begin{array}{l}\text { Region and } \\
\text { Metrics }\end{array}$ & $\begin{array}{l}\text { Healthy } \\
\text { Subjects } \\
(n=40)\end{array}$ & $\begin{array}{c}\text { Subjects } \\
\text { with DCM } \\
(n=58)\end{array}$ & $P$ Value & $\begin{array}{c}\text { Diagnostic } \\
\text { Accuracy } \\
\text { (AUC) }\end{array}$ \\
\hline \multicolumn{5}{|l|}{ Rostral } \\
\hline $\mathrm{CSA}\left(\mathrm{mm}^{2}\right)$ & $78.5 \pm 8.0$ & $70.9 \pm 10.4$ & $9 \times 10^{-5}$ & 0.722 \\
\hline FA & $0.725 \pm 0.036$ & $0.687 \pm 0.063$ & $2 \times 10^{-4}$ & 0.692 \\
\hline MTR & $52.7 \pm 2.4$ & $51.2 \pm 3.4$ & .01 & 0.648 \\
\hline T2*WI WM/GM & $0.848 \pm 0.031^{\mathrm{b}}$ & $0.884 \pm 0.034^{b}$ & $8 \times 10^{-7 b}$ & $0.775^{b}$ \\
\hline \multicolumn{5}{|l|}{$\mathrm{MCL} / \mathrm{C} 4-5$} \\
\hline $\mathrm{CSA}\left(\mathrm{mm}^{2}\right)$ & $76.2 \pm 10.4^{b}$ & $50.8 \pm 18.1^{b}$ & $1 \times 10^{-13 b}$ & $0.890^{\mathrm{b}}$ \\
\hline FA & $0.652 \pm 0.048$ & $0.553 \pm 0.094$ & $2 \times 10^{-9}$ & 0.813 \\
\hline MTR & $49.9 \pm 2.9$ & $47.6 \pm 3.8$ & .001 & 0.698 \\
\hline T2*WI WM/GM & $0.850 \pm 0.022$ & $0.899 \pm 0.038$ & $1 \times 10^{-11}$ & 0.860 \\
\hline \multicolumn{5}{|l|}{ Caudal } \\
\hline $\mathrm{CSA}\left(\mathrm{mm}^{2}\right)$ & $63.7 \pm 9.1$ & $60.1 \pm 10.9$ & .08 & 0.585 \\
\hline FA & $0.599 \pm 0.050$ & $0.552 \pm 0.060$ & $2 \times 10^{-4}$ & $0.724^{b}$ \\
\hline MTR & $46.2 \pm 3.8$ & $46.4 \pm 5.1$ & .85 & 0.515 \\
\hline T2*WI WM/GM & $0.862 \pm 0.047^{b}$ & $0.903 \pm 0.053^{b}$ & $1 \times 10^{-4 b}$ & 0.721 \\
\hline
\end{tabular}

increased T2*WI WM/GM (rostral: $P=8 \times 10^{-7}$; MCL: $P=1 \times$ $10^{-11}$; caudal: $P=1 \times 10^{-4}$ ), decreased FA (rostral: $P=2 \times$ $10^{-4}$; MCL: $P=2 \times 10^{-9}$; caudal: $\left.P=2 \times 10^{-4}\right)$, and decreased MTR (rostral: $P=.01$; MCL: $P=.001$ ). Patients with DCM also showed a trend toward decreased caudal CSA $(P=.08)$. All differences remained significant compared with age-matched healthy subjects, and caudal CSA became borderline significant $(P=.05)$. The strongest cross-correlations were found between the same metrics at different levels (eg, rostral and caudal CSA: $r=0.77$ ) (Fig 3). Cross-correlations were relatively strong between MCL metrics $(0.44-0.57)$ but weaker at rostral and caudal levels.

\section{Diagnostic Accuracy}

MCL CSA showed the highest diagnostic accuracy with AUC = 0.890 , outperforming other metrics at MCL: T2*WI WM/GM (0.860), FA (0.813), and MTR (0.698) (Table 2). At the rostral and caudal levels, T2*WI WM/GM showed better discrimination than other metrics with AUC $=0.775$ and 0.721 , respectively. T2WI hyperintensity was present in 37/58 (64\%) of subjects with DCM and $0 / 40$ healthy subjects, with AUC $=0.640$. Multivariate analysis with logistic regression achieved $\mathrm{AUC}=0.954$, retaining rostral T2*WI WM/GM $(P=.02)$, MCL FA $(P=.12)$, MCL CSA $(P=.14)$, and T2WI signal change $(P=.71)$.

\section{Correlation with Global and Focal Impairment}

The strongest univariate correlate with the mJOA score was MCL CSA $(r=0.66)$ (Table 3$)$. This was stronger than MCL T2*WI $\mathrm{WM} / \mathrm{GM}(r=-0.59), \mathrm{FA}(r=0.54)$, and MTR $(r=0.43)$. At the rostral and caudal levels, T2*WI WM/GM showed the strongest correlation with the mJOA score ( $r=-0.52,-0.36$, respectively). Multiple linear regression for the mJOA score found a good fit $\left(R^{2}=0.59\right.$, adjusted $\left.R^{2}=0.55, P=8 \times 10^{-13}\right)$, with rostral $\mathrm{T} 2{ }^{\star} \mathrm{WI}$ WM/GM showing the strongest relationship $(P=.01)$, followed by rostral MTR $(P=.02)$, T2WI signal change $(P=.02)$, caudal CSA $(P=.05)$, caudal FA $(P=.27)$, MCL CSA $(P=.34)$, and MCL FA $(P=.44)$. The strongest correlate with UE motor and sensory scores was rostral $\mathrm{T} 2{ }^{\star} \mathrm{WI}$ WM/GM, extracted from the ipsilateral lateral corticospinal tract $(r=$ $\left.-0.45, P=7 \times 10^{-11}\right)$ and fasciculus cuneatus $\left(r=-0.49, P=4 \times 10^{-13}\right)$, respectively.

\section{Effects of T2WI Hyperintensity}

Subjects with DCM with T2WI with hyperintensity had lower mJOA scores than those with T2WI without hyperintensity (13.6 versus 15.2, $P=.005)$ and higher MCL T2*WI WM/GM (0.905 versus $0.886, P=.07)$. When we analyzed all 98 subjects, 2-way ANOVA found significant independent relationships with the mJOA scores for $\mathrm{T} 2{ }^{*} \mathrm{WI}$ WM/GM $(P=.01)$ and T2WI signal change $(P=.001)$, while the interaction term was nonsignificant $(P=.55)$, sug- 


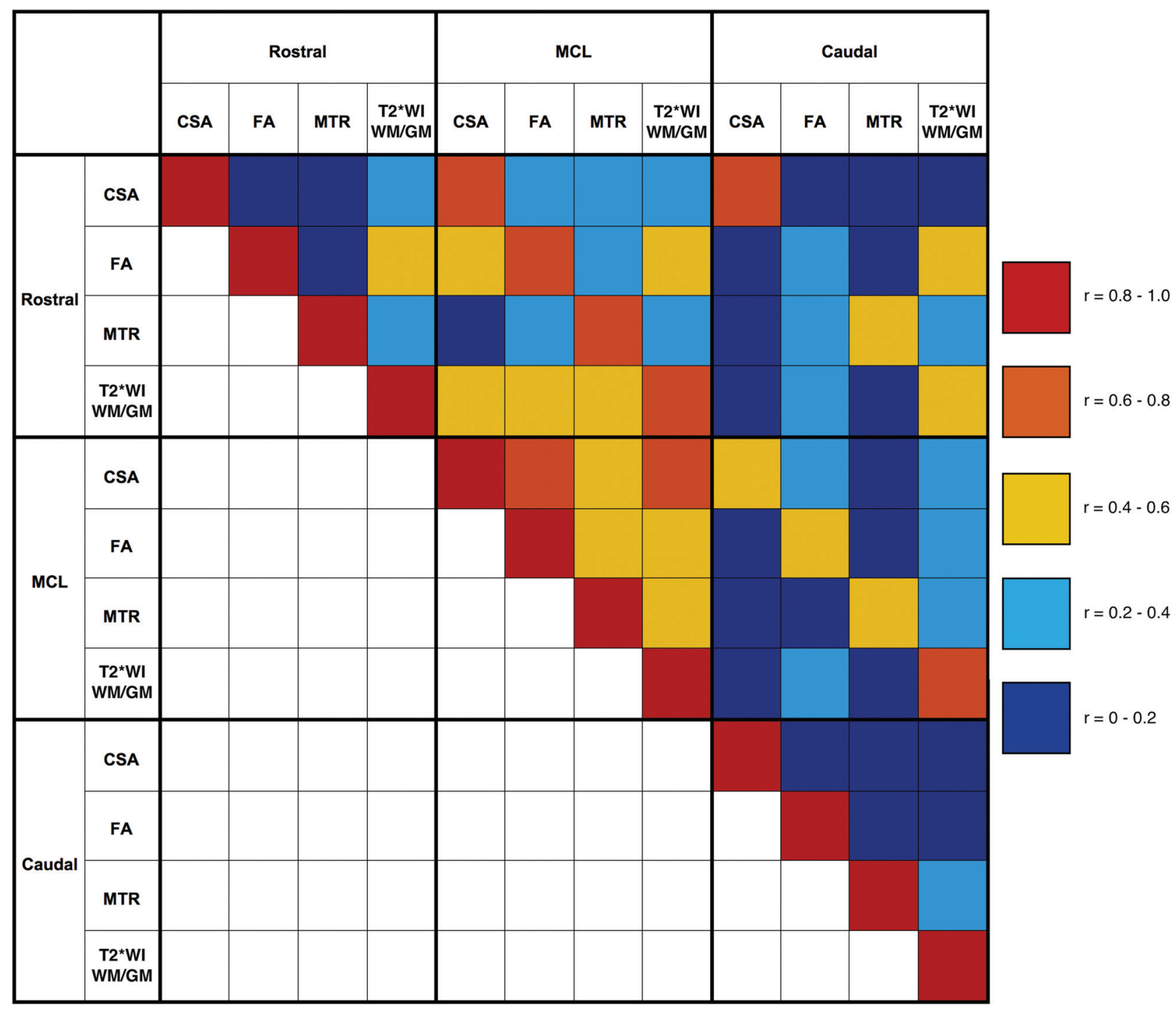

FIG 3. Correlation matrix for MR imaging metrics. Pearson correlation coefficients calculated between MR imaging metrics at rostral (Cl-C3), MCL (or C4-5 in healthy subjects), and caudal (C6-7) levels are color-coded to represent the degree of cross-correlation by using data from all 98 subjects.

Table 3: Correlation with clinical measures ${ }^{a}$

\begin{tabular}{lccc}
\hline $\begin{array}{c}\text { Region and } \\
\text { MRI Metrics }\end{array}$ & $\begin{array}{c}\text { mJOA Score } \\
(\boldsymbol{n}=98)\end{array}$ & $\begin{array}{c}\text { UE Motor Score } \\
(\boldsymbol{n}=196)\end{array}$ & $\begin{array}{c}\text { UE Sensory Score } \\
(\boldsymbol{n}=196)\end{array}$ \\
\hline Rostral & & - & - \\
CSA & $0.44\left(6 \times 10^{-6}\right)$ & - & - \\
FA & $0.37\left(2 \times 10^{-4}\right)$ & $0.20(0.006)$ & $0.26\left(3 \times 10^{-4}\right)$ \\
MTR & $0.35\left(5 \times 10^{-4}\right)$ & $0.22(0.002)$ & $0.11(0.13)$ \\
T2*WI WM/GM & $-0.52\left(5 \times 10^{-8}\right)^{\mathrm{b}}$ & $-0.45\left(7 \times 10^{-11}\right)^{\mathrm{b}}$ & $-0.49\left(4 \times 10^{-13}\right)^{\mathrm{b}}$ \\
MCL/C4-5 & & - & - \\
CSA & $0.66\left(2 \times 10^{-13}\right)^{\mathrm{b}}$ & - & $0.40\left(1 \times 10^{-8}\right)$ \\
FA & $0.54\left(2 \times 10^{-8}\right)$ & $0.36\left(5 \times 10^{-7}\right)^{\mathrm{b}}$ & $0.05(0.48)$ \\
MTR & $0.43\left(1 \times 10^{-5}\right)$ & $0.14(0.04)$ & $-0.43\left(8 \times 10^{-10}\right)^{\mathrm{b}}$ \\
T2*WI WM/GM & $-0.59\left(7 \times 10^{-10}\right)$ & $-0.33\left(3 \times 10^{-6}\right)$ & - \\
Caudal & & - & $0.05(0.49)$ \\
CSA & $0.27(0.007)$ & $0.09(0.20)$ & $0.05(0.51)$ \\
FA & $0.35(0.001)$ & $0.12(0.11)$ & $-0.25\left(6 \times 10^{-4}\right)^{\mathrm{b}}$ \\
MTR & $0.02(0.83)$ & $-0.17(0.01)^{\mathrm{b}}$ & - \\
T2*WI WM/GM & $-0.36\left(3 \times 10^{-4}\right)^{\mathrm{b}}$ &
\end{tabular}

${ }^{a}$ mJOA is analyzed against FA, MTR, and T2*WI WM/GM extracted from total WM and SC CSA. UE motor score and UE sensory score are analyzed with respect to non-CSA metrics extracted from the ipsilateral, lateral corticospinal tract and fasciculus cuneatus, respectively. Pearson coefficients are shown with $P$ values in parentheses.

${ }^{\mathrm{b}}$ Strongest correlations with clinical measures for each region. gesting that T2WI hyperintensity does not impact the performance of $\mathrm{T} 2{ }^{\star} \mathrm{WI}$ WM/GM. The within-group correlation between MCL T2*WI WM/GM and mJOA scores was slightly higher among subjects without hyperintensity $(r=$ -0.43 ) than among subjects with T2WI hyperintensity $(r=-0.36)$ (Table 4$)$.

\section{DISCUSSION}

\section{Summary of Findings}

All 4 qMRI metrics analyzed in this study demonstrated significant results in terms of group differences and clinical correlations, which was encouraging given the predominance of subjects with mild DCM in our cohort. MCL CSA outperformed other measures in all univariate analyses; this result is not surprising because this measure of spinal 
Table 4: Analysis of $\mathrm{T}^{*} \mathrm{WI}$ WM/GM and T2WI signal change ${ }^{\mathrm{a}}$

\begin{tabular}{lccc}
\hline \multicolumn{1}{c}{ Measure } & T2WI- $(\boldsymbol{n}=61)$ & T2WI+ $(\boldsymbol{n}=37)$ & $\boldsymbol{P}$ Value \\
\hline mJOA & $17.0 \pm 1.6$ & $13.6 \pm 2.8$ & $7 \times 10^{-9}$ \\
MCL T2*WI WM/GM & $0.862 \pm 0.033$ & $0.905 \pm 0.037$ & $2 \times 10^{-7}$ \\
MCL T2*WI WM/GM $\sim$ mJOA & $-0.43\left(9 \times 10^{-4}\right)$ & $-0.36(0.03)$ & \\
\hline
\end{tabular}

${ }^{a}$ The entire cohort (including subjects with DCM and healthy subjects) is divided into subjects with and without T2WI hyperintensity, denoted T2WI+ and T2WI-, respectively. Mean \pm SD are reported. T2*WI WM/GM is extracted from the MCL (subjects with DCM) or C4-5 (healthy subjects), and Pearson correlation coefficients between $\mathrm{mJOA}$ and T2*WI WM/GM within each signal change group are shown ( $P$ values in parentheses).

cord compression reflects the primary mechanism of tissue injury in DCM. Cord compression causes ischemia that often represents partially reversible neurologic impairment, ${ }^{30}$ whereas atrophy of the SC (rostral or caudal compression) suggests axonal loss or demyelination, which is more likely to be permanent. ${ }^{12}$ MCL CSA has been previously demonstrated to correlate well with severity in DCM, ${ }^{31}$ and atrophy measurement has also proved useful in $\mathrm{DCM}^{12}$ and MS. ${ }^{4-6}$ However, MCL CSA does not account for motion-related dynamic injury, which is also believed to be an important mechanism of tissue injury in DCM, ${ }^{31}$ suggesting that this metric may be better used in conjunction with other measures that directly interrogate microstructural changes. FA showed strong group differences and moderate correlations with impairment, but diagnostic accuracy was modest. These findings are all consistent with those in the previous literature. ${ }^{3,9-14}$ MTR results were relatively weak, which is consistent with findings in prior studies in MS, ${ }^{16,17}$ but differ from results seen in chronic SCI. ${ }^{15}$

We are not aware of published reports using MTR in patients with DCM. The T2*WI WM/GM signal ratio showed the strongest results at the rostral and caudal levels, and rostral $\mathrm{T}{ }^{\star} \mathrm{WI}$ $\mathrm{WM} / \mathrm{GM}$ was the strongest independent variable in multivariate models for diagnosis and correlation with the mJOA score. T2*WI WM/GM also demonstrated superior performance over FA and MTR in almost every comparison. The encouraging findings for $\mathrm{T} 2{ }^{*} \mathrm{WI} \mathrm{WM} / \mathrm{GM}$ indicate that this novel biomarker is a relatively accurate measure of WM injury, with particularly strong results in multivariate models. T2*WI WM/GM also shows better reliability, compared with FA and MTR, with our techniques. ${ }^{7}$ In comparison with DTI and MT techniques, T2*WI had fewer excluded sections, required less imaging time, and involved less postprocessing, suggesting that this biomarker is well-suited for clinical use.

Unfortunately, all qMRI metrics failed to show diagnostic accuracy (AUC) of $>90 \%$ and provided only moderate clinical correlations, indicating somewhat limited utility when used individually. However, our protocol produced 10 measures of tissue injury that are relatively independent, enabling multivariate use to strengthen their accuracy. This was evident in the logistic regression model that achieved $>95 \%$ diagnostic accuracy, and the linear regression model for the mJOA score that had much higher adjusted $R^{2}$ than univariate measures. Overall, our results demonstrate that $\mathrm{T} 2{ }^{*} \mathrm{WI}$ WM/GM performs well in comparison with established biomarkers, and our multiparametric approach has the potential to overcome the limitations of individual qMRI measures.
T2*WI WM/GM: A Novel Biomarker of WM Injury

$\mathrm{T} 2^{\star} \mathrm{WI}$ is available from all major MR imaging vendors, including the GE Healthcare multiecho recombined gradient-echo, Siemens multiecho data image combination, Philips Healthcare multiecho fast-field gradient echo, and Hitachi ADAGE (Additive Arrangement Gradient Echo) sequences, though differences may exist between implementations, and cross-vendor validation is needed. ${ }^{32}$ Our investigation of the T2*WI WM/GM signal intensity ratio follows from previous findings that $\mathrm{T} 2{ }^{\star} \mathrm{WI}$ detects WM injury by exhibiting hyperintensity. In one study, a pattern consistent with Wallerian degeneration of the fasciculus gracilis could be visualized rostrally following a cervical SC needle injury. ${ }^{19}$ Another study found hyperintensity in the bilateral lateral corticospinal tracts in a patient with amyotrophic lateral sclerosis, related to the degeneration of descending upper motor neurons. ${ }^{20}$ In our data, a small number of subjects with DCM also exhibited focal T2*WI hyperintensity of the dorsal columns extending through all images rostral to compression, consistent with Wallerian degeneration (Fig 2). However, most patients with DCM showed only loss of gray-white contrast, which is somewhat akin to the diagnosis of acute ischemic stroke on brain CT. However, T2*WI signal intensity is a relative value that varies considerably between subjects, requiring normalization. Although GM may also experience injury in DCM, we found that using GM signal intensity as a reference produced more consistent results than CSF due to variable CSF signal (A.R.M. et al, unpublished data, 2017). Furthermore, T2*WI WM/GM appears to be stable in the context of T2WI hyperintensity; this feature is commonly encountered in DCM, showing no significant interaction (effect modification) and minimal impact on clinical correlations.

The calculation of the WM/GM signal-intensity ratio is easily and accurately performed by using automated template-based analysis. ${ }^{29}$ The pathophysiologic processes that underlie $\mathrm{T} 2{ }^{\star} \mathrm{WI}$ hyperintensity include demyelination, gliosis, increased calcium concentration, and nonheme iron stored in ferritin, but signal intensity also depends on water content and local concentration of deoxyhemoglobin (used in blood oxygen level-dependent fMRI). ${ }^{33-37}$ Thus, T2*WI WM/GM is somewhat nonspecific, reflecting several microstructural features. The moderate crosscorrelations observed between $\mathrm{T} 2{ }^{*} \mathrm{WI} \mathrm{WM} / \mathrm{GM}$ and other metrics did not reveal a clear pattern because these findings may simply be explained by multiple pathologic processes occurring simultaneously. Histopathologic studies are necessary to fully understand exactly what SC microstructural changes are detected by $\mathrm{T} 2{ }^{\star}$ WI WM/GM compared with other measures, and further research is needed to determine its performance in other pathologies. However, its simplicity, sensitivity, and excellent reliability suggest that it could be a very useful imaging biomarker.

\section{ROIs}

The strongest results for each metric were found at the MCL in this study, with the exception of rostral T2*WI WM/GM for multivariate analyses and tract-specific correlations. This finding highlights a major challenge to using quantitative MR imaging in 
DCM because the compressed region has potential bias related to distorted anatomy (leading to inaccurate registration to the template) and increased susceptibility artifacts. This challenge was partially mitigated by averaging MCL metrics over 3 sections, with sections above and below MCL often showing no compression. However, results from our reliability study showed a trend toward diminished reliability for FA, MTR, and T2*WI WM/GM at the MCL. ${ }^{7}$ It was encouraging to also find strong results rostrally for T2*WI WM/GM, which has been previously reported for FA. ${ }^{14,38}$ This finding has important clinical implications because this region avoids the aforementioned issues and can be used for postoperative assessment rostral to metallic implants in most patients with DCM. This region is also potentially useful for the prediction of outcomes in acute SCI, with a postoperative scan in the days to weeks following early surgical decompression. ${ }^{38}$ The caudal region consistently showed the weakest results, likely due to respiratory motion, susceptibility artifacts from the lungs, and increased partial volume effects due to the angle between sections and the SC (in subjects with irreducible cervical lordosis). Despite these issues, T2*WI WM/GM and FA showed some utility in this region. Metrics extracted from individual WM tracts showed significant correlations with focal neurologic deficits, particularly at the rostral and MCL levels, indicating that our quantitative analysis identifies focal tissue injury. However, correlations with motor/sensory scores were modest, potentially because of the small number of voxels included in metric calculations but also because clinical impairment can also result from GM injury, nerve root compression (radiculopathy), and pain.

\section{Future Directions: Clinical Translation of Quantitative Spinal Cord MR Imaging}

At present, SC qMRI has yet to achieve clinical adoption due to challenges with the portability of acquisitions, cumbersome analysis, and modest results in diagnostic accuracy and clinical correlations. However, our multiparametric approach with simple methods and automated analysis is designed to address each of these issues and be suitable for clinical use. We anticipate that the first clinical application of these techniques could be the development of more sensitive diagnostic tools. A diagnostic tool that can directly detect tissue injury could have a major impact in DCM, in which patients sometimes show minimal symptoms that cannot be definitely attributed to the SC by clinical and electrophysiological examinations. Furthermore, many older individuals have spinal cord compression without neurologic dysfunction, ${ }^{39}$ indicating that anatomic imaging alone is insufficient. Our approach may also prove useful for monitoring patients with DCM for progression of tissue injury by using serial qMRI examinations. Patients with mild DCM are often managed nonoperatively with periodic clinical assessments, but symptoms are highly subjective and mechanisms of behavioral adaptation and neuroplasticity may mask subtle deterioration. Finally, effort has been made to predict outcomes by using qMRI in DCM and other clinical populations, ${ }^{3,14}$ but this effort has yet to show great success, possibly because outcomes depend on factors that extend beyond the current state of tissue injury. However, if qMRI techniques can differentiate between reversible and permanent injury by quantifying specific microstructural changes (eg, demyelination versus axonal loss), enhanced outcome prediction may also be possible. Future studies should be directed at investigating each of these exciting potential applications.

\section{Limitations}

Clinical assessments used in this study are somewhat coarse (mJOA score, sensory score) and subjective (mJOA score, motor score), potentially limiting the strength of correlations. T1weighted imaging was not performed in this study, and the effect of T1WI hypointensity on T2*WI WM/GM has not been characterized. We aimed to minimize bias by using automated analysis, but almost all DCM datasets required manual correction of segmentation. Other DTI metrics were not analyzed due to an a priori decision to focus on FA, due to its consistent results in previous studies. ${ }^{3}$ The validity of MR imaging metrics for 3 patients with metallic implants is unknown, but quantitative results distant from the hardware appeared to be consistent with those in other subjects.

\section{CONCLUSIONS}

$\mathrm{T} 2{ }^{\star} \mathrm{WI} \mathrm{WM} / \mathrm{GM}$ is a novel biomarker of SC WM degeneration that shows good diagnostic accuracy and correlation with clinical features of DCM, warranting further investigation. This biomarker has strong potential for clinical translation, particularly in multivariate approaches that combine quantitative measures of SC injury. Such measures have the potential to provide more sensitive diagnosis of mild cord injury, monitoring of disease progression or recovery, and prediction of outcomes in DCM and other spinal pathologies.

\section{ACKNOWLEDGMENTS}

This research received funding support from Rick Hansen Institute, as part of the Riluzole in Spinal Cord Injury Study (RISCIS), which is also supported by AOSpine North America, AOSpine International SCI Knowledge Forum, and the North American Clinical Trials Network (NACTN) of the Christopher and Dana Reeve Foundation. This research also received support from the Dezwirek Foundation, the Sherman Clinical Research Unit, and the Gerald and Tootsie Halbert Chair in Spinal Cord Research. Dr. Martin received post-doctoral fellowship support from Canadian Institutes of Health Research. We would also like to thank all subjects for their participation and acknowledge MRI Technologists Keith Ta and Eugen Hlasny for their key contributions, and several students, research coordinators, and administrators that assisted with this research.

Disclosures: Allan R. Martin—RELATED: Grant: Rick Hansen Institute, AOSpine North America, AOSpine International SCI Knowledge Forum, the North American Clinical Trials Network of the Christopher and Dana Reeve Foundation, the DeZwirek Foundation, the Sherman Clinical Research Unit, and the Gerald and Tootsie Halbert Chair in Spinal Cord Research*; Dr. Martin received post-doctoral Fellowship funding from Canadian Institutes of Health Research (CIHR) that included $\$ 50,000$ (CDN) annual salary support and $\$ 5,000$ annual research allowance that enabled this research. Sukhvinder Kalsi-Ryan-UNRELATED: Consultancy: Neural Outcomes; Royalties: GRASSP, Version 1.0. *Money paid to the institution.

\section{REFERENCES}

1. Stroman PW, Wheeler-Kingshott C, Bacon M, et al. The current state-of-the-art of spinal cord imaging: methods. Neuroimage 2014; 84:1070-81 CrossRef Medline 
2. Wheeler-Kingshott CA, Stroman PW, Schwab JM, et al. The current state-of-the-art of spinal cord imaging: applications. Neuroimage 2014;84:1082-93 CrossRef Medline

3. Martin AR, Aleksanderek I, Cohen-Adad J, et al. Translating stateof-the-art spinal cord MRI techniques to clinical use: a systematic review of clinical studies utilizing DTI, MT, MWF, MRS, and fMRI. Neuroimage Clin 2016;10:192-238 CrossRef Medline

4. Oh J, Seigo M, Saidha S, et al. Spinal cord normalization in multiple sclerosis. J Neuroimaging 2014;24:577-84 CrossRef Medline

5. Kearney H, Yiannakas MC, Abdel-Aziz K, et al. Improved MRI quantification of spinal cord atrophy in multiple sclerosis. J Magn Reson Imaging 2014;39:617-23 CrossRef Medline

6. Kearney H, Altmann DR, Samson RS, et al. Cervical cord lesion load is associated with disability independently from atrophy in MS. Neurology 2015;84:367-73 CrossRef Medline

7. Martin AR, De Leener B, Cohen-Adad J, et al. Clinically feasible microstructural MRI to quantify cervical spinal cord tissue injury using DTI, MT, and T2*-weighted imaging: assessment of normative data and reliability. AJNR Am J Neuroradiol 2017 Apr 20. [Epub ahead of print] CrossRef Medline

8. Kato F, Yukawa Y, Suda K, et al. Normal morphology, age-related changes and abnormal findings of the cervical spine, part II: magnetic resonance imaging of over 1,200 asymptomatic subjects. Eur Spine J 2012;21:1499-507 CrossRef Medline

9. Uda T, Takami T, Tsuyuguchi N, et al. Assessment of cervical spondylotic myelopathy using diffusion tensor magnetic resonance imaging parameter at 3.0 Tesla. Spine (Phila Pa 1976) 2013;38:407-14 CrossRef Medline

10. Budzik JF, Balbi V, Le Thuc V, et al. Diffusion tensor imaging and fibre tracking in cervical spondylotic myelopathy. Eur Radiol 2011; 21:426-33 CrossRef Medline

11. Ellingson BM, Salamon N, Grinstead JW, et al. Diffusion tensor imaging predicts functional impairment in mild-to-moderate cervical spondylotic myelopathy. Spine J 2014;14:2589-97 CrossRef Medline

12. Grabher P, Mohammadi S, Trachsler A, et al. Voxel-based analysis of grey and white matter degeneration in cervical spondylotic myelopathy. Sci Rep 2016;6:24636 CrossRef Medline

13. Jones JG, Cen SY, Lebel RM, et al. Diffusion tensor imaging correlates with the clinical assessment of disease severity in cervical spondylotic myelopathy and predicts outcome following surgery. AJNR Am J Neuroradiol 2013;34:471-78 CrossRef Medline

14. Wen CY, Cui JL, Liu HS, et al. Is diffusion anisotropy a biomarker for disease severity and surgical prognosis of cervical spondylotic myelopathy? Radiology 2014;270:197-204 CrossRef Medline

15. Cohen-Adad J, El Mendili MM, Lehéricy S, et al. Demyelination and degeneration in the injured human spinal cord detected with diffusion and magnetization transfer MRI. Neuroimage 2011;55:1024-33 CrossRef Medline

16. Oh J, Saidha S, Chen M, et al. Spinal cord quantitative MRI discriminates between disability levels in multiple sclerosis. Neurology 2013;80:540-47 CrossRef Medline

17. Oh J, Zackowski K, Chen M, et al. Multiparametric MRI correlates of sensorimotor function in the spinal cord in multiple sclerosis. Mult Scler 2013;19:427-35 CrossRef Medline

18. Yiannakas MC, Kearney H, Samson RS, et al. Feasibility of grey matter and white matter segmentation of the upper cervical cord in vivo: a pilot study with application to magnetisation transfer measurements. Neuroimage 2012;63:1054-59 CrossRef Medline

19. Cohen-Adad J, Buchbinder B, Oaklander AL. Cervical spinal cord injection of epidural corticosteroids: comprehensive longitudinal study including multiparametric magnetic resonance imaging. Pain 2012;153:2292-99 CrossRef Medline

20. Cohen-Adad J, Zhao W, Keil B, et al. 7-T MRI of the spinal cord can detect lateral corticospinal tract abnormality in amyotrophic lateral sclerosis. Muscle Nerve 2013;47:760-62 CrossRef Medline

21. Taso M, Girard OM, Duhamel G, et al. Tract-specific and age-related variations of the spinal cord microstructure: a multi-parametric MRI study using diffusion tensor imaging (DTI) and inhomogeneous magnetization transfer (ihMT). NMR Biomed 2016;29: 817-32 CrossRef Medline

22. Samson RS, Levy S, Schneider T, et al. ZOOM or non-ZOOM? Assessing spinal cord diffusion tensor imaging protocols for multicentre studies. PLoS One 2016;11:e155557 CrossRef Medline

23. Kerkovsky M, Bednarik J, Dusek L, et al. Magnetic resonance diffusion tensor imaging in patients with cervical spondylotic spinal cord compression: correlations between clinical and electrophysiological findings. Spine (Phila Pa 1976) 2012;37:48-56 CrossRef Medline

24. Smith SA, Jones CK, Gifford A, et al. Reproducibility of tract-specific magnetization transfer and diffusion tensor imaging in the cervical spinal cord at 3 Tesla. NMR Biomed 2010;23:207-17 CrossRef Medline

25. Samson RS, Ciccarelli O, Kachramanoglou C, et al. Tissue- and column-specific measurements from multi-parameter mapping of the human cervical spinal cord at 3 T. NMR Biomed 2013;26:1823-30 CrossRef Medline

26. Fehlings MG, Wilson JR, Kopjar B, et al. Efficacy and safety of surgical decompression in patients with cervical spondylotic myelopathy: results of the AOSpine North America prospective multi-center study. J Bone Joint Surg Am 2013;95:1651-58 CrossRef Medline

27. Nouri A, Martin AR, Mikulis D, et al. Magnetic resonance imaging assessment of degenerative cervical myelopathy: a review of structural changes and measurement techniques. Neurosurg Focus 2016; 40:E5 CrossRef Medline

28. Kirshblum SC, Burns SP, Biering-Sorensen F, et al. International standards for neurological classification of spinal cord injury (revised 2011). J Spinal Cord Med 2011;34:535-46 CrossRef Medline

29. De Leener B, Lévy S, Dupont SM, et al. SCT: Spinal Cord Toolbox, an open-source software for processing spinal cord MRI data. Neuroimage 2017;145(pt A):24-43 CrossRef Medline

30. Tetreault LA, Karpova A, Fehlings MG. Predictors of outcome in patients with degenerative cervical spondylotic myelopathy undergoing surgical treatment: results of a systematic review. Eur Spine J 2015;24(suppl 2):236-51 CrossRef Medline

31. Nouri A, Tetreault L, Zamorano JJ, et al. Role of magnetic resonance imaging in predicting surgical outcome in patients with cervical spondylotic myelopathy. Spine 2015;40:171-78 CrossRef Medline

32. White ML, Zhang Y, Healey K. Cervical spinal cord multiple sclerosis: evaluation with 2D multi-echo recombined gradient echo MR imaging. J Spinal Cord Med 2011;34:93-98 CrossRef Medline

33. Cohen-Adad J. What can we learn from $\mathrm{T}^{\star}$ maps of the cortex? Neuroimage 2014;93(pt 2):189-200 CrossRef Medline

34. Lee J, Shmueli K, Kang BT, et al. The contribution of myelin to magnetic susceptibility-weighted contrasts in high-field MRI of the brain. Neuroimage 2012;59:3967-75 CrossRef Medline

35. Fukunaga M, Li TQ, van Gelderen P, et al. Layer-specific variation of iron content in cerebral cortex as a source of MRI contrast. Proc Natl Acad Sci U S A 2010;107:3834-39 CrossRef Medline

36. Haacke EM, Cheng NY, House MJ, et al. Imaging iron stores in the brain using magnetic resonance imaging. Magn Reson Imaging 2005; 23:1-25 CrossRef Medline

37. Marques JP, Maddage R, Mlynarik V, et al. On the origin of the MR image phase contrast: an in vivo MR microscopy study of the rat brain at 14.1 T. Neuroimage 2009;46:345-52 CrossRef Medline

38. Vedantam A, Eckardt G, Wang MC, et al. Clinical correlates of high cervical fractional anisotropy in acute cervical spinal cord injury. World Neurosurg 2015;83:824-28 CrossRef Medline

39. Wilson JR, Barry S, Fischer DJ, et al. Frequency, timing, and predictors of neurological dysfunction in the nonmyelopathic patient with cervical spinal cord compression, canal stenosis, and/or ossification of the posterior longitudinal ligament. Spine 2013;38: S37-54 CrossRef Medline 